\title{
Src tyrosine kinase is crucial for potassium channel function in human pulmonary
} arteries

\author{
Chandran Nagaraj*,\#, Bi Tang\#, Zoltán Bálint*, Malgorzata Wygrecka", \\ Andelko Hrzenjak*, Grazyna Kwapiszewska*, Elvira Stacher ${ }^{+}$, Joerg Lindenmann ${ }^{\S}$, \\ E. Kenneth Weir ${ }^{f}$, Horst Olschewski ${ }^{\#}$ and Andrea Olschewski ${ }^{\star * * *}$
}

ABSTRACT: The potassium channel TWIK-related acid sensitive potassium (TASK)-1 channel, together with other potassium channels, controls the low resting tone of pulmonary arteries. The Src family tyrosine kinase (SrcTK) may control potassium channel function in human pulmonary artery smooth muscle cells (hPASMCs) in response to changes in oxygen tension and the clinical use of a SrcTK inhibitor has resulted in partly reversible pulmonary hypertension.

This study aimed to determine the role of SrcTK in hypoxia-induced inhibition of potassium channels in hPASMCs.

We show that SrcTK is co-localised with the TASK-1 channel. Inhibition of SrcTK decreases potassium current density and results in considerable depolarisation, while activation of SrcTK increases potassium current in patch-clamp recordings. Moderate hypoxia and the SrcTK inhibitor decrease the tyrosine phosphorylation state of the TASK-1 channel. Hypoxia also decreases the level of phospho-SrcTK (tyr419) and reduces the co-localisation of the TASK-1 channel and phospho-SrcTK. Corresponding to this, hypoxia reduces TASK-1 currents before but not after SrcTK inhibition and, in the isolated perfused mouse lung, SrcTK inhibitors increase pulmonary arterial pressure.

We propose that the SrcTK is a crucial factor controlling potassium channels, acting as a cofactor for setting a negative resting membrane potential in hPASMCs and a low resting pulmonary vascular tone.

KEYWORDS: Pulmonary artery pressure, resting membrane potential, Src tyrosine kinase, TWIKrelated acid sensitive potassium-1 channel

$\mathrm{n}$ the pulmonary artery, vascular tone is regulated in part by the resting membrane potential of the pulmonary artery smooth muscle cells (PASMCs). As in all excitable cells, potassium channels are primary candidates for the regulation of the resting membrane potential by maintaining the resting membrane potential close to the potassium equilibrium potential. Activation or inhibition of the potassium channels results in hyperpolarisation or depolarisation of the cell membrane, leading to initiation of vasodilation and vasoconstriction, respectively. At least four classes of potassium channels have been identified in PASMCs [1]. The background or leak potassium-selective channels are exceptional amongst the potassium channels, as their activity is not controlled by voltage and they may be considered as the main contributor to the resting membrane potential and input resistance in primary human pulmonary artery smooth muscle cells (hPASMCs) [2, 3].

Src family tyrosine kinase (SrcTK) is a member of the nonreceptor tyrosine kinase family participating in a wide range of cellular signalling and functions [4]. c-SrcTK is targeted to the plasma membrane due to a myristylated N-terminal region. The SH1 domain of SrcTK contains the phosphorylation site Tyr419, which is required for full c-SrcTK activation [5]. This mechanism allows c-SrcTK to interact with ion channels to modulate their properties $[6,7]$. Inactivation of human c-SrcTK occurs when its
AFFILIATIONS

*Ludwig Boltzmann Institute for Lung Vascular Research, Medical University of Graz,

"Division of Pulmonology, Dept of Internal Medicine, Medical University of Graz,

${ }^{+}$Institute of Pathology, Medical University of Graz,

${ }^{\S}$ Division of Thoracic and Hyperbaric Surgery, Dept of Surgery, Medical University of Graz, and

**Experimental Anesthesiology, Dept of Anaesthesia and Intensive Care Medicine, Medical University of Graz, Graz, Austria.

"Dept of Biochemistry University of Giessen Lung Center, Giessen, Germany.

fDept of Medicine, VA Medical Center and University of Minnesota, Minneapolis, MN, USA.

\section{CORRESPONDENCE}

A. Olschewski, Ludwig Boltzmann Institute for Lung Vascular Research and Experimental Anesthesiology Dept of Anaesthesia and Intensive Care Medicine, Medical University of Graz, Stiftingtalstr. 24, A-8036 Graz, Austria

E-mail: andrea.olschewski@ medunigraz.at

Received:

Dec 062011

Accepted after revision:

April 032012

First published online:

April 202012 
C-terminal Tyr530 is phosphorylated; this then binds to the $\mathrm{SH} 2$ domain. Crystallographic studies have shown that interactions between the C-terminus and the $\mathrm{SH} 2$ domain, and between the kinase domain and the $\mathrm{SH} 3$ domain, cause the c-SrcTK molecule to assume a closed configuration that covers the kinase domain and reduces its potential for substrate interaction [5]. The Cterminus of the background TWIK-related acid sensitive potassium (TASK)-1 channel contains possible phosphorylation sites for tyrosine and serine kinases (fig. S1a). If potassium channels are normally activated by SrcTK, reversal of this mechanism could explain the increased pulmonary vascular tone observed after SrcTK inhibition by dasatinib treatment [8].

In the present study, we investigated the functional role of SrcTK on potassium channel function and membrane potential. We studied hypoxic inhibition of TASK-1 channels in hPASMCs to define the specific role of SrcTK. We demonstrate that specific inhibition of SrcTK results in the inhibition of TASK-1 and other potassium channels and causes membrane depolarisation. In addition, hypoxia decreases active phospho-SrcTK co-localisation with TASK-1 channels in the membrane, while activation of SrcTK increases TASK-1 current and this activated current is inhibited by moderate hypoxia. This suggests that potassium channel function is critically dependent on SrcTK, thereby explaining why SrcTK inhibition might cause life-threatening pulmonary vasoconstriction. This is the first report to demonstrate the functional role of SrcTK in the regulation of resting membrane potential, in the hypoxic inhibition of TASK-1 channels in primary hPASMCs and, finally, in pulmonary vascular tone.

\section{METHODS}

The study protocol for tissue donation was approved by the Institutional Review Board of the Medical University of Graz, Graz, Austria, and is in accordance with the national law and the guidelines on Good Clinical Practice/International Conference on Harmonization (www.ich.org/). Written informed consent was obtained from each patient if appropriate. All animal experiments were approved by the Institutional Animal Care and Use Committee (BMWF, Austria and Medical University of Graz, Graz, Austria).

\section{Preparation of primary hPASMCs and cell culture}

Primary smooth muscle cells were isolated from human resistance pulmonary arteries from patients $(n=30)$ undergoing lung surgery for lung cancer without a history of pulmonary vascular disease or arterial hypoxaemia, or unused donor lungs harvested for lung transplantation. In the case of obtaining pulmonary arteries from patients with lung cancer, only arteries that were a distance of $\geqslant 5 \mathrm{~cm}$ from the cancer tissue were used. For details, see the online supplementary material.

\section{Electrophysiology}

The whole-cell patch-clamp technique on hPASMCs was used as previously described to measure the resting membrane potential under current clamp and macroscopic potassium currents under voltage clamp [3]. Detailed description of the protocols and solutions are given in the online supplementary material.
The data were stored and analysed with commercially available pCLAMP 9.0 software (Axon Instruments, Foster City, CA, USA).

\section{Calcium measurements}

The fluorescent dye fluo-4-AM was used for detection for detection of changes in intracellular calcium in hPAMSCs. Detailed description of the protocols and solutions are given in the online supplementary material.

The acquired images were stored and subsequently processed offline with TillVision software (Till Photonics, Munich, Germany).

\section{Transfection of small interfering RNA against c-Src, Fyn and TASK-1}

Small interfering RNAs (siRNAs) against c-Src (siC-Src), Fyn (siFyn) and TASK-1 (siTASK-1) were commercially synthesised (Eurogentec, Seraing, Belgium). For details, see the online supplementary material. As a negative control, nonsilencing RNA (nsRNA), which does not target any human gene product, was used. The hPASMCs were grown on coverslips or in sixwell plates, and annealed siRNA was transfected using Effectene transfection reagent (Qiagen, Hilden, Germany). Gene knock-down was checked by quantitative RT-PCR using the RNA extracted (RNeasy; Qiagen) from the transfected cells.

RNA levels, live-cell calcium and electrophysiological measurements were performed $48-56 \mathrm{~h}$ post-transfection. To assess the efficiency of the siRNA transfection, fluorescein isothiocyanate (FITC)-conjugated siRNA was used. Only FITC-positive cells were used for electrophysiological studies. In addition, siTASK-1 transfection was functionally controlled by superfusion of the cells with a bath solution adjusted to a $\mathrm{pH}$ of 8.3.

\section{RT-PCR}

Detailed description is provided in the online supplementary material.

\section{Quantitative RT-PCR}

Quantitative RT-PCR was performed to check the expression of c-Src, Fyn and TASK-1 in hPASMCs. For more details, see the online supplementary material.

\section{Co-immunoprecipitation, immunoblotting and immuno-co-} localisation

For details, see the online supplementary material.

\section{Hypoxic treatment of hPASMCs}

The effect of hypoxia in the patch clamp and calcium-imaging studies was studied by switching between normoxic and hypoxic perfusate reservoirs. A detailed description is provided in the online supplementary material.

\section{Isolated, perfused and ventilated mouse lungs}

Lungs from adult C57BL/6 mice (Harlan Laboratories, Inc., Indianapolis, IN, USA) were removed from the chest under deep anaesthaesia and articial ventilation, and perfused with Krebs' Henseleit buffer $\left(\mathrm{NaCl} 120 \mathrm{mmol} \cdot \mathrm{L}^{-1}, \mathrm{KCl} 4.3 \mathrm{mmol} \cdot \mathrm{L}^{-1}\right.$, $\mathrm{KH}_{2} \mathrm{PO}_{4} 1.1 \mathrm{mmol} \cdot \mathrm{L}^{-1}, \mathrm{CaCl}_{2} 2.4 \mathrm{mmol} \cdot \mathrm{L}^{-1}, \mathrm{MgCl}_{2} 1.3 \mathrm{mmol} \cdot \mathrm{L}^{-1}$ and glucose $13.3 \mathrm{mmol} \cdot \mathrm{L}^{-1}$, as well as $5 \%$ (weight/volume) hydroxyethylamylopectin (molecular weight 200,000 Da)). The lungs were mounted in a water-heated chamber that allowed 
for negative pressure ventilation with a gas containing $5.3 \%$ $\mathrm{CO}_{2}$ and $21.0 \% \mathrm{O}_{2}$, and balanced with $\mathrm{N}_{2}$. An initial steady state period of $15 \mathrm{~min}$ (with Krebs' Henseleit buffer at a flow rate $1 \mathrm{~mL} \cdot \mathrm{min}^{-1}$ ) was taken as baseline. For a detailed description, see the online supplementary material. 4-amino-5-(4-chlorophenyl)7-(t-butyl)pyrazolo[3,4-d]pyrimidine (PP2; $30 \mu \mathrm{M})$, 4-amino-7phenylpyrazol[3,4-d]pyrimidine (PP3; $30 \mu \mathrm{M})$ or dasatinib $(100 \mu \mathrm{M})$ was added into the buffer for a period of $15 \mathrm{~min} . \Delta P \mathrm{pa}$ indicates the change in pulmonary arterial pressure $(P$ pa $)$ after application of PP2, PP3 or dasatinib.

\section{Solutions and chemicals}

PP2 and PP3 were purchased from Sigma Chemical Company (St Louis, MO, USA). Src activator peptide was from Santa Cruz Biotechnology Inc. (Santa Cruz, CA, USA). All drugs were dissolved in the experimental (bath) solution, except for PP2, PP3 and dasatinib. They were dissolved in dimethyl sulphoxide (DMSO). At this concentration, the vehicle alone had no effect on ion current, resting membrane potential or $\mathrm{Ppa}$. The $\mathrm{pH}$ of solutions containing drugs was tested and corrected to eliminate potential $\mathrm{pH}$-induced effects using Krebs' Henseleit buffer. $\mathrm{NaHCO}_{3}$, used as a buffer, was adjusted to result in a constant $\mathrm{pH}$ of 7.37-7.40.

\section{Statistical analysis}

Numerical values are given as mean \pm SE of $n$ cells or measurements. Intergroup differences were assessed by a factorial ANOVA with post hoc analysis with Tukey's test, or unpaired and paired t-tests as appropriate. p-values $<0.05$ were considered significant.

\section{RESULTS}

\section{Expression of SrcTK in human lung and PASMCs}

To analyse the expression of SrcTK isoforms, c-Src, Lck, Lyn, Fyn, Yes and Frg, RT-PCR was performed on human lung tissue, human pulmonary artery and hPASMCs (fig. 1a). Our results demonstrate the presence of mRNA encoding all investigated isoforms in lung tissue. Primary hPASMCs expressed mRNA only for the isoforms c-Src, Fyn and Yes.

\section{SrcTK is co-localised with TASK-1 channels in hPASMC Co-immunoprecipitation of TASK-1 with SrcTK}

Immunoprecipitation was performed with the anti-SrcTK antibody or isotype control (immunoglobulin (Ig)G) from the hPASMC lysate. Later immunoblotting was performed either with anti-TASK-1 (fig. 1b, top panel) or with anti-ScrTK antibodies (fig. $1 \mathrm{~b}$, bottom panel), showing the direct binding of TASK-1 to SrcTK.

The localisation of the background TASK-1 channel and SrcTK were also visualised by confocal laser scanning microscopy in hPASMCs (fig. 1c). Immunofluorescent staining of TASK-1 and SrcTK revealed that TASK-1 and SrcTK are co-localised in the cell membrane. Staining was absent in negative controls when cells without exposure to primary antibody were imaged. The TASK-1 and SrcTK antibodies were evaluated by immunoblot (fig. S1b).

\section{SrcTK modulates TASK-1 channel activity in hPASMCs}

To further confirm the functional relevance of the SrcTK interaction with TASK-1 channels, we used pharmacological tools and siRNA techniques. Patch-clamp recordings of the noninactivating TASK-1 current on hPASMCs were made as described previously [3]. SrcTK increases TASK-1 channel activity as confirmed by silencing of c-Src and Fyn in primary hPASMCs, resulting in decreased current compared with control (fig. 2a and b). TASK-1 current density is significantly inhibited by treatments with the SrcTK inhibitor PP2 $\left(0.23 \pm 0.06 \mathrm{pA} \cdot \mathrm{pF}^{-1}, \mathrm{n}=12\right)$. Although PP2 is a potent and selective inhibitor of the Src family of protein tyrosine kinases, in order to investigate the role of the Src subtypes, siRNA against c-Src and Fyn was used. Transfection with siC-Src $\left(0.17 \pm 0.01 \mathrm{pA} \cdot \mathrm{pF}^{-1}, \mathrm{n}=4\right)$ or siFyn $\left(0.20 \pm 0.02 \mathrm{pA} \cdot \mathrm{pF}^{-1}, \mathrm{n}=8\right)$ significantly inhibited TASK-1 current density compared with control $\left(0.67 \pm 0.1 \mathrm{pA} \cdot \mathrm{pF}^{-1}, \mathrm{n}=13\right)$. As a control, $\mathrm{PP} 3$, the inactive analogue of the c-Src inhibitor PP2 and transfection with nsRNA was applied. The treatment with PP3 $\left(0.58 \pm 0.02 \mathrm{pA} \cdot \mathrm{pF}^{-1}, \mathrm{n}=4\right)$ or transfection with nsRNA $\left(0.62 \pm 01 \mathrm{pA} \cdot \mathrm{pF}^{-1}, \mathrm{n}=8\right)$ does not significantly affect TASK-1 current (fig. 2c). The relative degree of knock-down of c-Src and Fyn by their respective siRNA was confirmed by quantitative RT-PCR (fig. S2).

\section{SrcTK inhibition depolarises hPASMCs}

TASK-1 channels are active at resting membrane potential and set the negative resting membrane potential in PASMCs, as we have previously demonstrated by siRNA treatment in primary hPASMCs. As the SrcTK inhibition also decreases the TASK-1 current (fig. 2c), the physiological role of SrcTK for the resting membrane potential of primary hPASMCs was further investigated. Electrophysiological measurements carried out in hPASMCs showed a significant depolarisation after treatment with PP2 $(-24.1 \pm 1.3 \mathrm{mV}, \mathrm{n}=39)$ or transfection with siC-Src $(-19.6 \pm 1.5 \mathrm{mV}, \mathrm{n}=14)$ or with siFyn $(-20 \pm 1.1 \mathrm{mV}, \mathrm{n}=14)$ compared either with control $(-44.3 \pm 1.6 \mathrm{mV}, \mathrm{n}=36)$ or with cells either treated with the inactive analogue of PP2, PP3 $(-43.4 \pm 1.5 \mathrm{mV}, \mathrm{n}=4)$ or transfected with nsRNA $(-43.6 \pm 1.5 \mathrm{mV}$, $\mathrm{n}=18$ ) (fig. $2 \mathrm{~d}$ ). Taken together, these data further strengthen the role of TASK-1 and the importance of SrcTK in setting the negative resting membrane potential in primary PASMCs.

\section{Hypoxic regulation of SrcTK in hPASMCs}

Hypoxia causes membrane depolarisation in hPASMCs, partly due to TASK-1 channel inhibition. Silencing of SrcTK decreases TASK-1 current leading to membrane depolarisation. Activity of SrcTK is determined by the phosphorylation state of SrcTK at Tyr419. Therefore, we investigated the phosphorylated (active; fig. 3a, top panel) and nonphosphorylated (inactive; fig. 3a, middle panel) state of SrcTK in hPASMCs at different timepoints under hypoxia (30 min of normoxia or $0,1,5,10,15,20$ and $30 \mathrm{~min}$ of hypoxia). Next, the co-localisation of the background TASK-1 channel and phospho-SrcTK was examined in hPASMCs under normoxia and after 15 min hypoxia, and results were visualised by confocal laser scanning microscopy (fig. 3b). The lower panel of figure $3 b$ clearly shows the reduced phospho-SrcTK staining in hypoxia. Figure $3 c$ shows that hypoxia, as well as application of the SrcTK-inhibitor PP2, decrease the tyrosine-phosphorylation state of TASK-1 channels, whereas PP3, the inactive analogue of PP2, does not change the TASK-1 phosphorylation. Figure $3 \mathrm{c}$ shows the unchanged total level of TASK-1 under different experimental conditions.

Next, the functional role of SrcTK for the hypoxic inhibition of TASK-1 channels was examined. Intracellular dialysis with Src 
a)

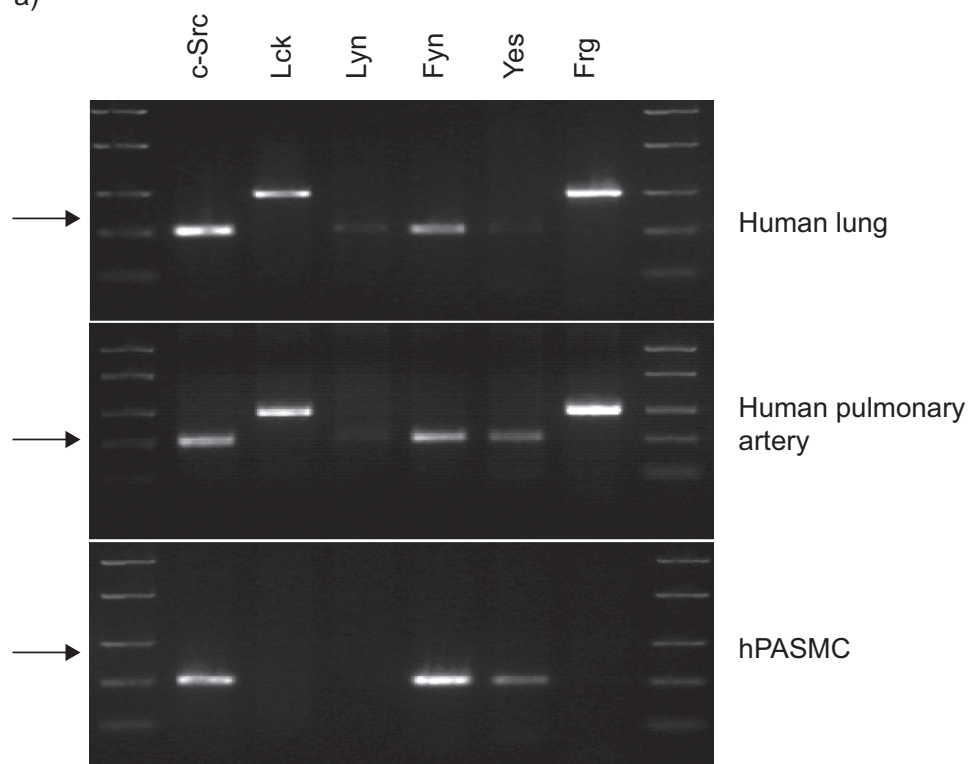

c)

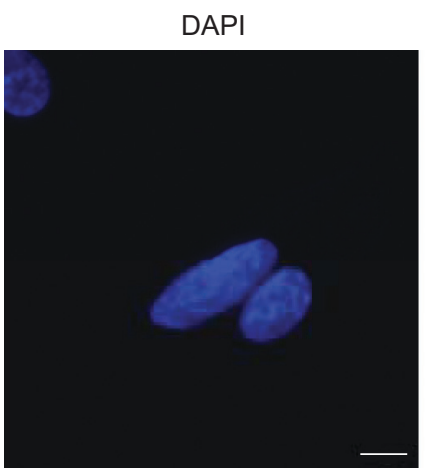

SrcTK

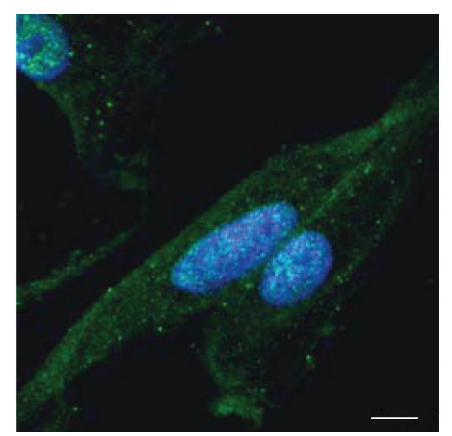

b)
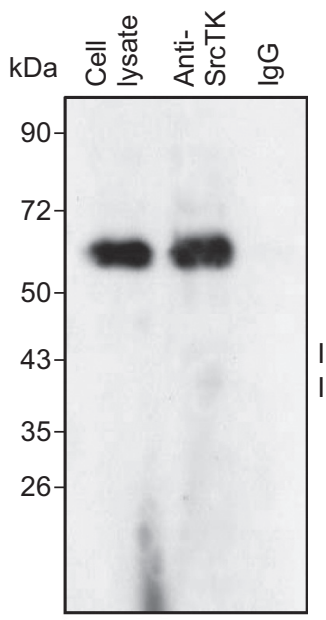

IP: SrcTK

IB: TASK-1

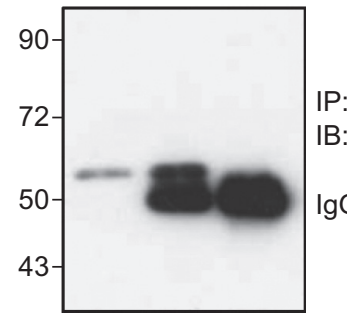

IP: SrcTK

IB: SrcTK

$\lg G$

Merged

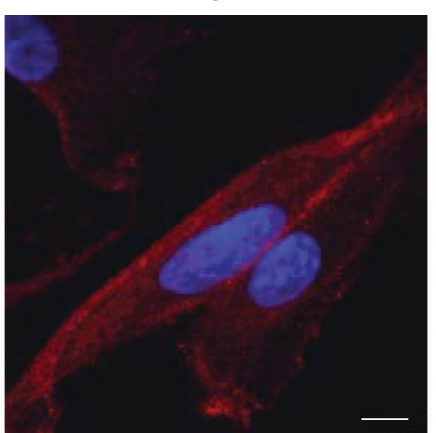

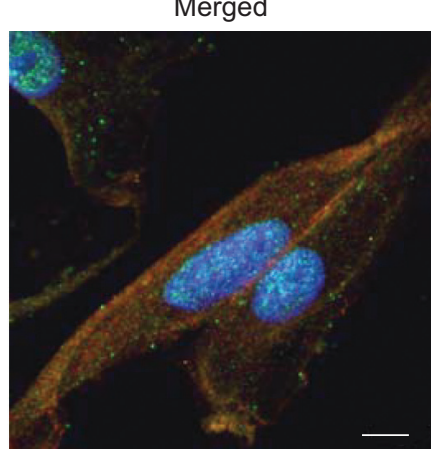

FIGURE 1. Src family tyrosine kinase (SrcTK) is co-localised with TWIK-related acid sensitive potassium (TASK)-1 channels in human pulmonary artery smooth muscle cells (hPASMCs). a) RT-PCR screening for SrcTK in RNA extracts from homogenised human lung tissue and primary hPASMCs. Representative gels illustrate mRNA expression of c-Src (204 bp), Lck (398 bp), Lyn (213 bp), Fyn (206 bp), Yes (202 bp) and Frg (402 bp). The arrows indicate 200 bp. Identical results were obtained with at least three preparations of RNA from different donor lungs and primary hPASMCs. b) Blot represents co-immunoprecipitation (IP) with anti-SrcTK, isotype control immunoglobulin (Ig)G and cell lysate, then immunoblotted (IB) for TASK-1 (upper panel) and SrcTK (lower panel). c) Fluorescent immunostainings indicate DAPI nuclear staining (blue), Src family kinases (red), TASK-1 channel (green) and an overlayed image (merged) in a single-plane confocal image of hPASMCs. Scale bar $=20 \mu \mathrm{m}$.

activator peptide (EPQYEEIPIYL) significantly increased the TASK-1 current density $\left(0.72 \pm 0.07 \mathrm{pA} \cdot \mathrm{pF}^{-1}, \mathrm{n}=8\right)$ compared with control $\left(0.51 \pm 0.05 \mathrm{pA} \cdot \mathrm{pF}^{-1}, \mathrm{n}=10\right.$; fig. $4 \mathrm{a}$ and $\left.\mathrm{b}\right)$. Hypoxia was able to inhibit both the control and the activated current. Figure 4c summarises the effects of SrcTK activator peptides and hypoxia. As the initial TASK-1 current was decreased by inhibiting SrcTK with blocker or small interfering (si)RNA (fig. 4e), hypoxia did not have any further effect on cells treated with PP2 or transfected with siRNA targeting c-Src (siC-Src) or siFyn. However, hypoxia was still able to inhibit the TASK-1 current in control, PP3-treated or nonsilencing RNA transfected hPASMCs (fig. 4d). Relative TASK-1 currents under hypoxia in hPASMCs are presented in figure $4 \mathrm{f}$.

TASK-1 channels can be modulated by different pathways, such as AMP-activated protein kinase (AMPK) [9], protein kinase (PK)A [3], PKC [10] and phospholipase (PL)C [11], in response to different agonist stimulations. In order to exclude the involvement of these pathways, we investigated the hypoxic inhibition of TASK-1 current using different inhibitors. None of these treatments affected control TASK- 1 current or its hypoxic inhibition (figs S3 and S4g), showing that AMPK, PKA, PKC and PLC are not involved.

\section{Impact of SrcTK on the hypoxia-induced increase of intracellular calcium in hPASMCs}

Hypoxia-induced rise in intracellular free calcium concentration $\left[\mathrm{Ca}^{2+}\right]_{\mathrm{i}}$ is an integral and characteristic property of hPASMCs that is directly linked to downstream signalling leading to vasoconstriction. The role of SrcTK in the hypoxia-induced $\left[\mathrm{Ca}^{2+}\right]_{\mathrm{i}}$ rise was further analysed in hPASMCs. Fluo4-loaded 

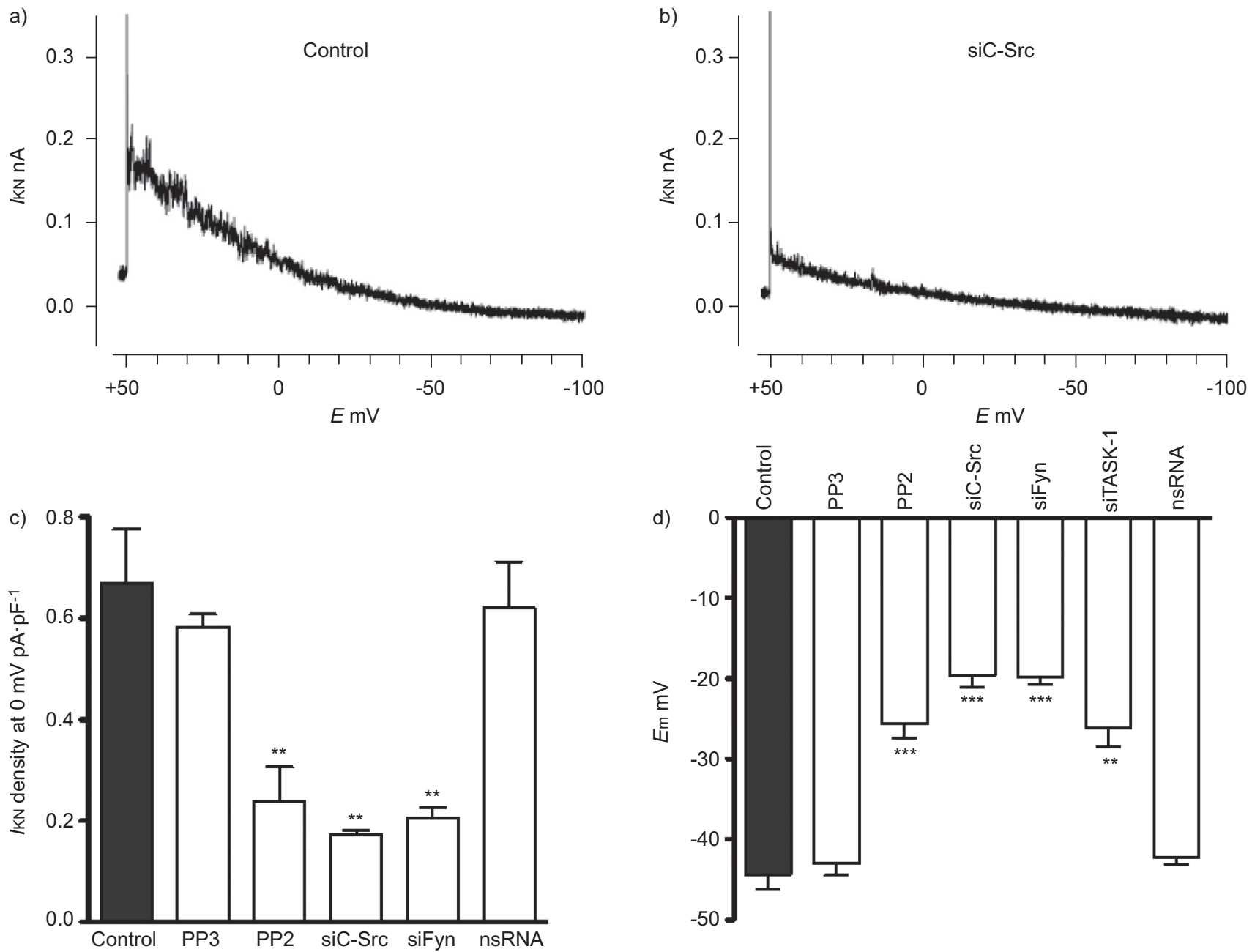

FIGURE 2. Src family tyrosine kinase (SrcTK) is crucial for TWIK-related acid sensitive potassium (TASK)-1 channel activity in primary human pulmonary artery smooth muscle cells (hPASMCs). Representative recordings of TASK-1 current (IKN) a) in control cells and b) in cells transfected with small-interfering RNA targeting c-Src (siC-Src). c) Histogram summarising the TASK-1 current density in primary hPASMCs. After treatment with Src family kinase inhibitor PP2 (1 $\mu$ M) or transfection with siC-Src and siFyn, the current density (IKN) is significantly reduced. There is no reduction of current density after transfection with nonsilencing (ns)RNA or after treatment with PP3 (1 $\mu \mathrm{M}$; inactive analogue of PP2). d) Histogram summarising the effects of SrcTK inhibition on resting membrane potential (Em) of hPASMCs. Significant depolarisation was observed when the cells were treated with PP2 $(1 \mu \mathrm{M})$ or transfected with siC-Src, siFyn or siTASK-1, but not after transfection with nsRNA and treatment with PP3. E: membrane potential. $* *: p<0.01$; ***: $p<0.001$ compared with control. Data in $c$ and d) are presented as mean \pm SE of $n$ cells or measurements.

hPASMCs were continuously monitored for changes in $\left[\mathrm{Ca}^{2+}\right]_{\mathrm{i}}$ under hypoxia (fig. 5a). Hypoxia significantly increased $\left[\mathrm{Ca}^{2+}\right]_{\mathrm{i}}$ in control cells $(0.54 \pm 0.04, \mathrm{n}=63)$, and in cells either treated with PP3 $(0.52 \pm 0.04, \mathrm{n}=17)$ or transfected with nsRNA $(0.45 \pm 0.06, \mathrm{n}=16)$. In contrast, the hypoxia-induced increase in $0.54 \pm 0.04$ was markedly attenuated after either treatment with PP2 $(0.18 \pm 0.01, \mathrm{n}=68)$ or transfection with siC-Src $(0.12 \pm 0.02, \mathrm{n}=18)$, siFyn $(0.12 \pm 0.02, \mathrm{n}=14)$ or siTASK-1 $(0.25 \pm 0.02, n=22$, respectively). Summarised results are presented in figure $5 b$.

\section{Inhibition of SrcTK attenuates voltage-gated and calcium- dependent potassium current in hPASMCs}

The impact of SrcTK inhibition on whole-cell potassium currents (voltage-gated $(\mathrm{Kv})$ and calcium-dependent $(\mathrm{KCa})$ potassium currents) was investigated in primary human PASMCs. Representative $\mathrm{K}_{\mathrm{v}}$ recordings in control hPASMCs (fig. 6a) and after treatment with siC-Src are presented in fig. $6 \mathrm{~b}$, showing that silencing of c-Src significantly decreases $\mathrm{K}_{v}$ current. Similar results were obtained from experiments recording KCa current in control (fig. 6d) and after silencing of c-Src (fig. 6e) in primary hPASMCs.

To further assess the role of SrcTK for activation of $\mathrm{K}_{\mathrm{v}}$ and $\mathrm{KCa}$ channels, the effect of PP2, PP3 and the treatment with siC-Src, siFyn or nsRNA was investigated and compared with the effect of hypoxia (fig. 6c). Treatment of hPASMCs with PP2 $\left(3.8 \pm 0.4 \mathrm{pA} \cdot \mathrm{pF}^{-1}, \mathrm{n}=16\right)$, or $\mathrm{siC}-\operatorname{Src}\left(2.9 \pm 0.3 \mathrm{pA} \cdot \mathrm{pF}^{-1}, \mathrm{n}=5\right)$ or siFyn $\left(3.1 \pm 0.3 \mathrm{pA} \cdot \mathrm{pF}^{-1}, \mathrm{n}=5\right)$ significantly decreased the $\mathrm{K}_{\mathrm{v}}$ current compared with the control $\left(8.9 \pm 0.8 \mathrm{pA} \cdot \mathrm{pF}^{-1}, \mathrm{n}=24\right.$, respectively). Hypoxia showed a similar effect (fig. 6c). Treatment with PP3 $\left(9.3 \pm 0.7 \mathrm{pA} \cdot \mathrm{pF}^{-1}, \mathrm{n}=4\right)$ or transfection with nsRNA $\left(7.8 \pm 0.4 \mathrm{pA} \cdot \mathrm{pF}^{-1}, \mathrm{n}=9\right)$ did not alter the current (fig. 6c). Similar results were observed when KCa current was recorded (fig. 6e and f). Only hypoxia $\left(2.3 \pm 0.5 \mathrm{pA} \cdot \mathrm{pF}^{-1}, \mathrm{n}=16\right)$, treatment 
a)
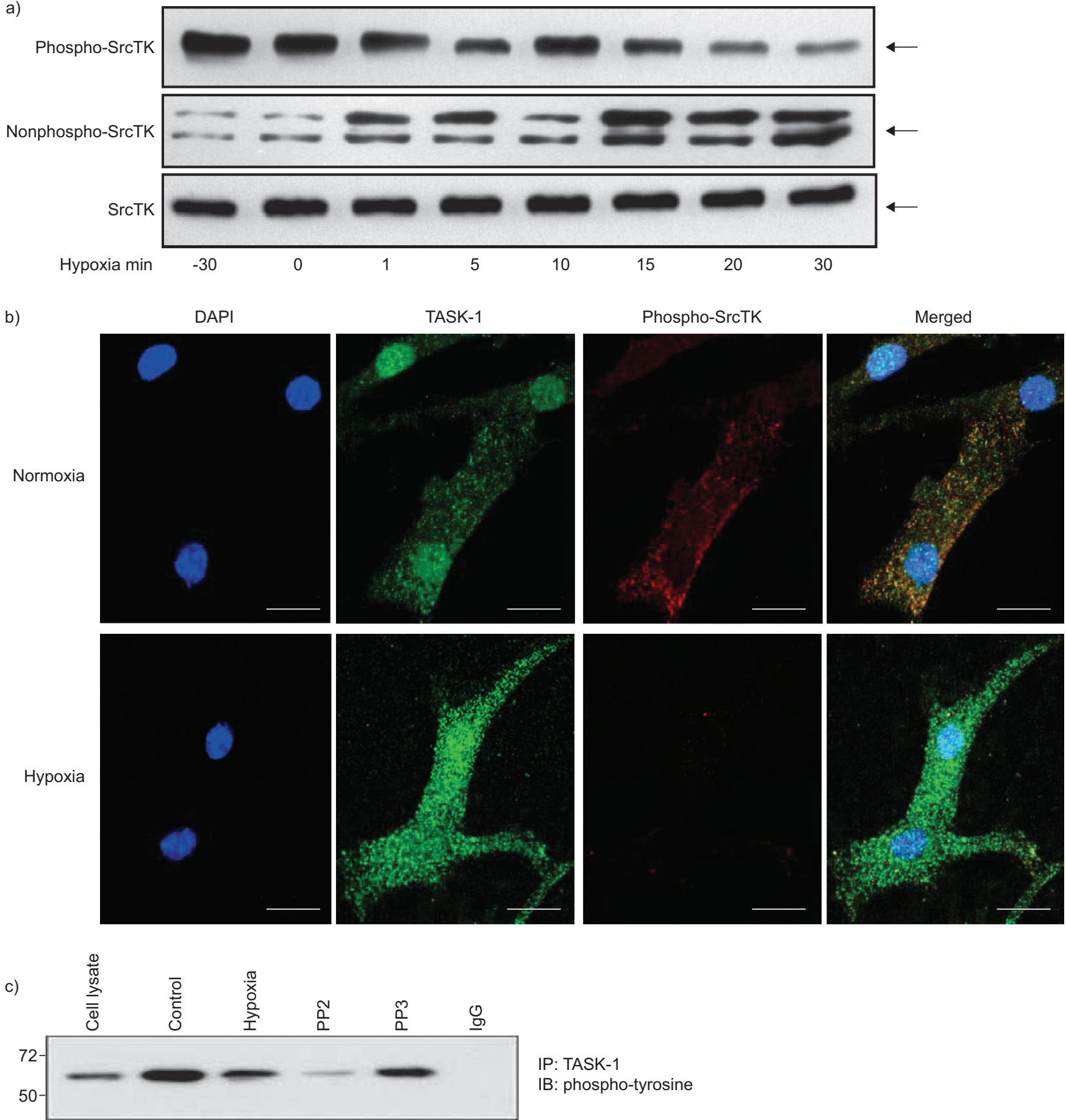

IP: TASK-1

IB: phospho-tyrosine

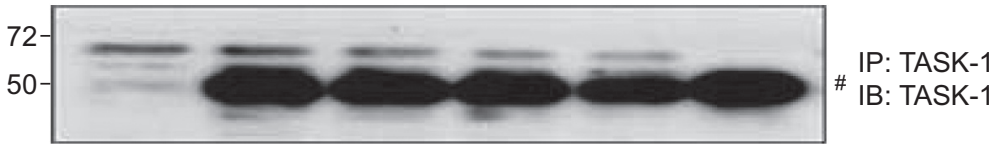

FIGURE 3. Effect of hypoxia on Src family tyrosine kinase (SrcTK) phosphorylation in primary human pulmonary artery smooth muscle cells (hPASMCs). a) Hypoxia decreased phospho-SrcTK (Tyr419) immunoreactivity $(60 \mathrm{kDa}$ ), whereas enhanced immunoreactivity was detected at the nonphospho-SrcTK (60-65 kDa) in a timedependent manner. Protein loading equivalence is shown by total SrcTK $(n=4)$ (where total SrcTk comprises phospho-SrcTK and nonphospho-SrcTK). b) An evident decrease of phospho-SrcTK Tyr419 (red) is shown after 15 min of hypoxia compared with normoxia in single-plane confocal images of hPASMC. Scale bars=20 $\mu \mathrm{m}$. C) Application of hypoxia or the SrcTK inhibitor PP2 decreased the tyrosine-phosphorylation state of TWIK-related acid sensitive potassium (TASK)-1 channel. The lower panel shows the unchanged total level of TASK-1 under different experimental conditions. IP: immunoprecipitation; IB: immunoblotting. "*: immunoglobulin (Ig)G. 

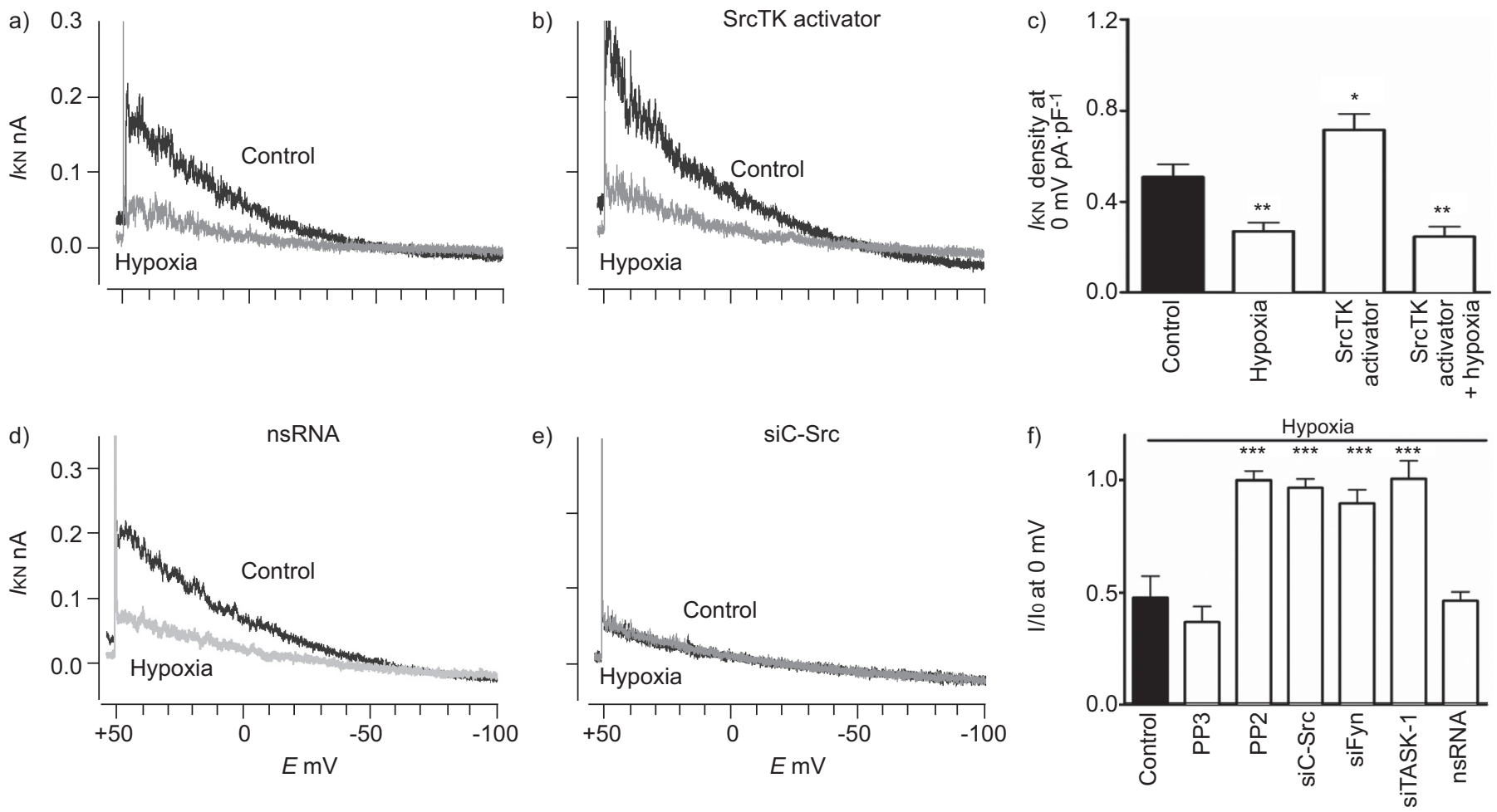

FIGURE 4. Src family tyrosine kinase (SrcTK) activator increases TWIK-related acid-sensitive potassium (TASK)-1 channel current and this effect is completely inhibited by hypoxia in primary human pulmonary artery smooth muscle cells (hPASMCs). Representative recordings of TASK-1 current (IKN) control and under hypoxia a) without or b) with SrcTK activator peptide (EPQYEEIPIYL; $1 \mathrm{mM}$ ) in the patch pipette. c) The effect of SrcTK activator peptide, which significantly increases the current density of TASK-1 current compared to control. This increased TASK-1 current is significantly inhibited by hypoxia. TASK-1 current recordings from cells transfected with either d) nonsilencing (ns)RNA or e) small-interfering RNA targeting c-Src (siC-Src). In the latter case, hypoxia could not further inhibit TASK-1 current. f) The lack of further hypoxia-induced TASK-1 current inhibition after treatment with PP2, and siC-Src, siFyn and siTASK-1. g) IKN density of TASK-1 channel in the presence of Ro-31-8220 (protein kinase (PK)C inhibitor), Gö6983 (PKC inhibitor), KT5720 (PKA inhibitor) and compound C (AMP-activated kinase inhibitor) under normoxia (control) and hypoxia in primary hPASMCs. 10: current under normoxia in every cell; $E$ : membrane potential. *: $p<0.05$; **: $p<0.01$; ***: $p<0.001$ compared with control.
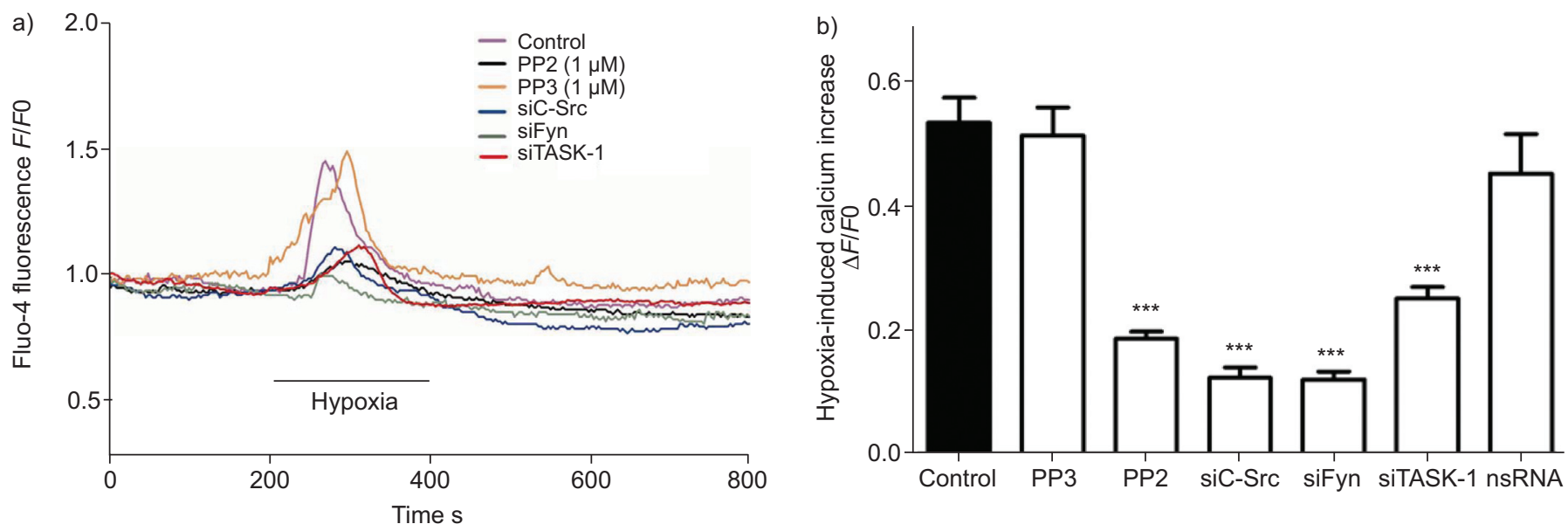

FIGURE 5. Inhibition of Src family tyrosine kinase attenuates hypoxia-induced increase in intracellular calcium concentration in primary human pulmonary artery smooth muscle cells (hPASMCs). a) Representative recordings of fluo-4 fluorescence ( $F$ ) after hypoxic challenge in control hPASMCs and in cells treated with PP2, PP3, small interfering RNA targeting c-Src (siC-Src) or siFyn. b) Hypoxia-induced intracellular calcium increase was decreased after treatment with PP2, siC-Src or siFyn, but not with nonsilencing (ns)RNA or PP3. Fo: fluorescence before challenge. TASK-1: TWIK-related acid sensitive potassium-1. ***: p<0.001 compared with control. 

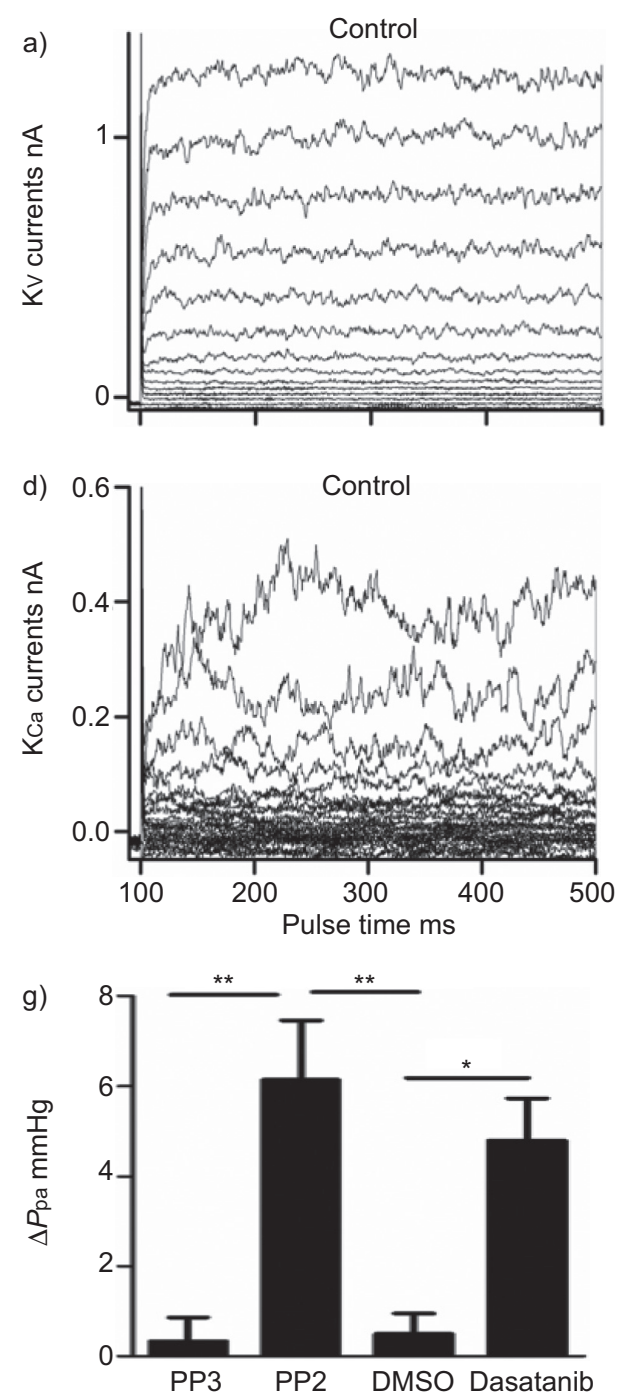

b)

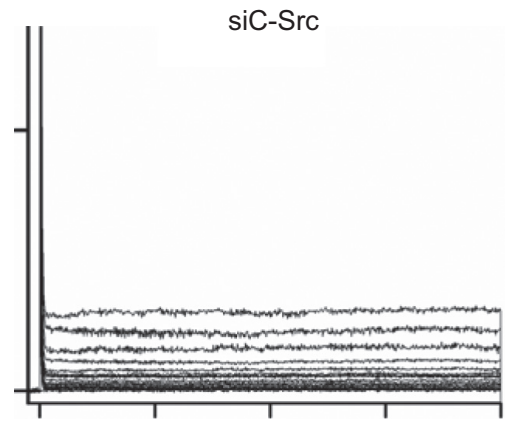

e)

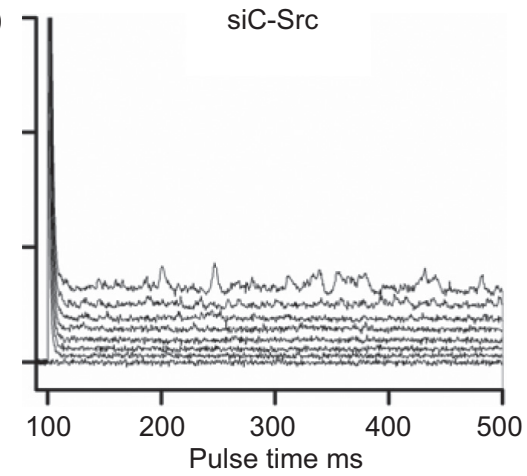

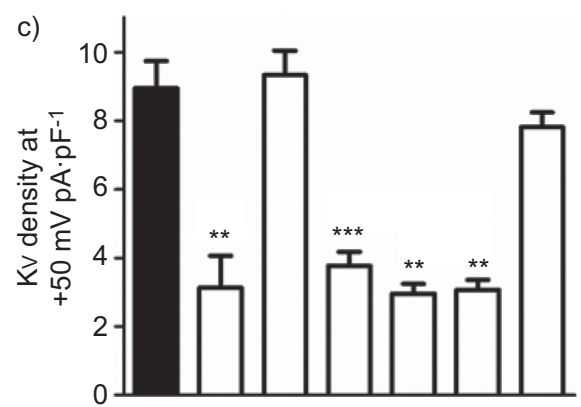

f)

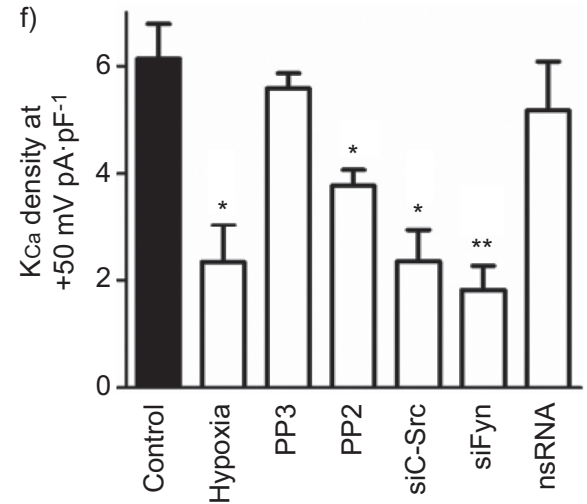

FIGURE 6. Inhibition of Src family tyrosine kinase decreases whole cell voltage-gated (Kv) and calcium-dependent $(\mathrm{KCa})$ potassium currents in primary human pulmonary artery smooth muscle cells and increases the pulmonary artery pressure $(P \mathrm{pa})$ in the isolated, perfused mouse lung model. a, d) Representative traces of $\mathrm{K}_{v}$ and $\mathrm{KCa}$ in control cells, and b, e) currents after treatment with small interfering RNA targeting c-Src (siC-Src). c, f) Histograms summarising the effects of hypoxia, PP3, PP2, siC-Src, siFyn or nonsilencing (ns)RNA) for $\mathrm{Kv}_{v}$ and $\mathrm{KCa}$ current densities. g) A histogram summarising change $(\Delta)$ in $P$ pa from isolated perfused mouse lungs in the presence of PP2, PP3, dimethyl sulphoxide (DMSO) and dasatinib. ${ }^{*}: p<0.05 ;{ }^{* *}: p<0.01 ; * * *: p<0.001$ compared with control.

with PP2 $\left(4 \pm 0.3 \mathrm{pA} \cdot \mathrm{pF}^{-1}, \mathrm{n}=17\right)$, siC-Src $\left(2.35 \pm 0.6 \mathrm{pA} \cdot \mathrm{pF}^{-1}\right.$, $\mathrm{n}=5)$ or siFyn $\left(1,81 \pm 0.4 \mathrm{pA} \cdot \mathrm{pF}^{-1}, \mathrm{n}=5\right)$ decreased the current compared with control $\left(5.7 \pm 0.6 \mathrm{pA} \cdot \mathrm{pF}^{-1}, \mathrm{n}=12\right)$, whereas PP3 $\left(5.58 \pm 0.3 \mathrm{pA} \cdot \mathrm{pF}^{-1}, \mathrm{n}=5\right)$ or $\operatorname{nsRA}\left(5.17 \pm 0.9 \mathrm{pA} \cdot \mathrm{pF}^{-1}, \mathrm{n}=8\right)$ had no significant effects (fig. 6f).

Pulmonary vasoconstriction in response to SrcTK inhibitors To depict the role of SrcTK in pulmonary vascular tone, we used isolated perfused mouse lungs, where PP2 showed a significant increase in $P$ pa $(6.3 \pm 1.3 \mathrm{mmHg}, \mathrm{n}=4)$ compared with the inactive analogue PP3 $(0.6 \pm 0.2 \mathrm{mmHg}, \mathrm{n}=4)$ and, interestingly, the second-line treatment of chronic myeloid leukaemia, dasatinib, which is also a potent inhibitor for SrcTK, showed a similar increase $(5.5 \pm 0.2 \mathrm{mmHg}, \mathrm{n}=3)$ in $P$ pa compared with the solvent DMSO $(0.4 \pm 0.4 \mathrm{mmHg}, \mathrm{n}=4)$. Figure $6 \mathrm{~g}$ shows the summarised $\triangle P$ pa before and after PP2, PP3, DMSO and dasatinib.

\section{DISCUSSION}

The main findings of this study are that: 1) two members of SrcTK family, c-Src and Fyn, are highly expressed in primary hPASMCs; 2) TASK-1 channels and SrcTK are co-localised in the plasma membrane of hPASMCs; 3) SrcTK is required for the activity of TASK-1 channels; 4) the inhibition of SrcTK depolarises hPASMCs; 5) hypoxia reduces the tyrosine phosphorylation level of TASK-1; 6) hypoxia reduces the active phosphorylated state of SrcTK and inhibits TASK-1 current facilitated by SrcTK activator; 7) SrcTK inhibition markedly attenuates the hypoxia-induced intracellular calcium rise; 8) $\mathrm{Kv}$ and KCa currents are reduced by SrcTK inhibition; and 9) inhibition of SrcTK by PP2 or dasatinib causes a substantial increase in the $P$ pa of isolated perfused mouse lungs.

The membrane potential of smooth muscle cells is an important factor in controlling pulmonary vascular tone. At rest, the membrane potential of PASMCs is approximately $-50 \mathrm{mV}[2,3$, 12]. This is maintained by potassium efflux from these cells through potassium channels. Agents that inhibit or activate pulmonary vascular smooth muscle cell potassium channels cause depolarisation or hyperpolarisation, respectively. The function, control and expression of potassium channels in pulmonary arteries is a matter of continued interest, because it is probable that a decrease in potassium channel expression, e.g. potassium voltage-gated channel, shaker-related subfamily, member 5 (Kv1.5) [13,14], or dysfunction of potassium channels gives rise to membrane depolarisation [15] and, together with an 


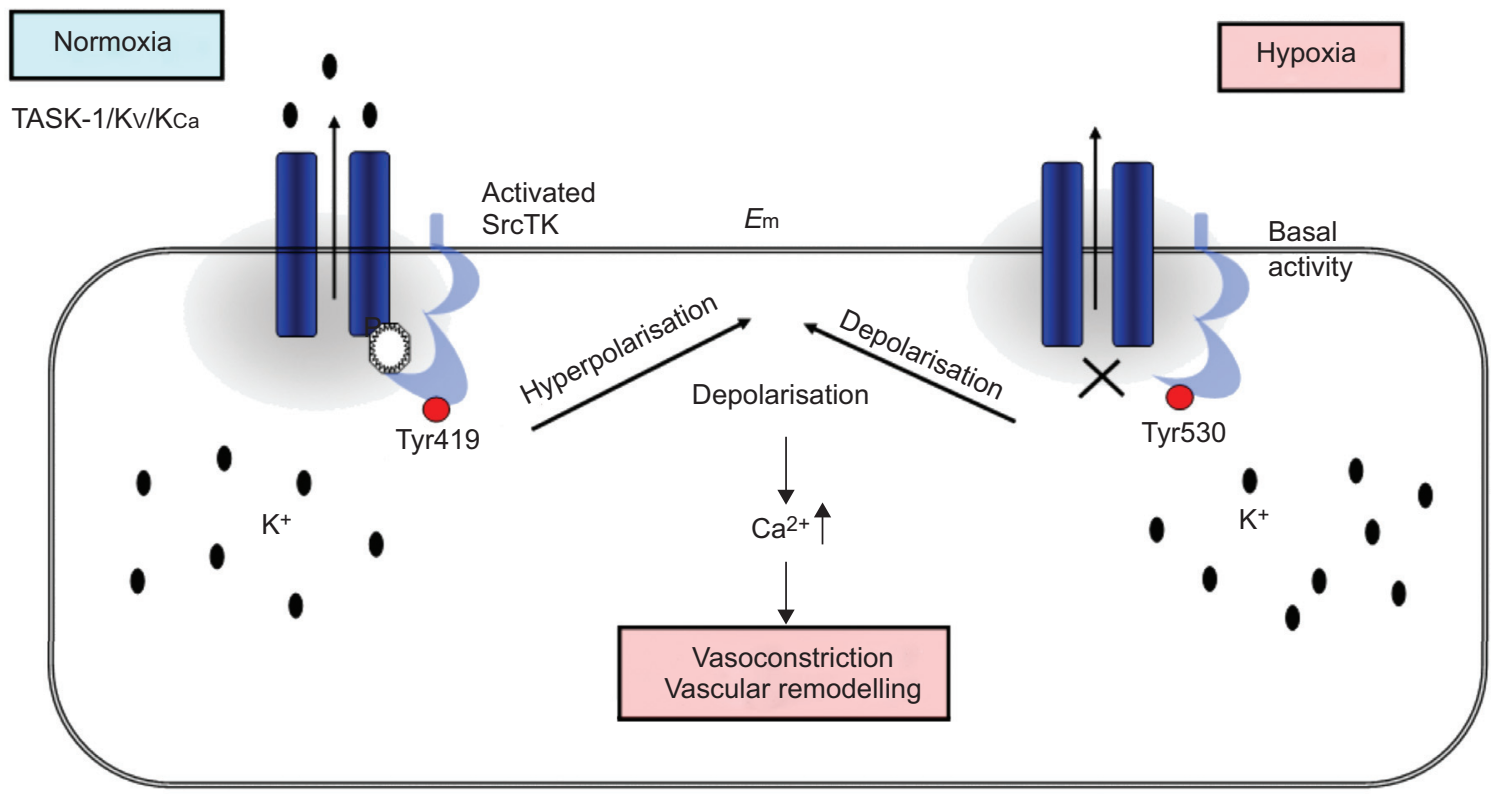

FIGURE 7. Scheme of the proposed interplay between TWIK-related acid sensitive potassium (TASK)-1 channel and c-Src in human pulmonary artery smooth muscle cells (hPASMCs). Under normoxia, the phospho-Src (active Src, phosphorylated at Tyr419) binds to TASK-1 channels resulting in functional TASK-1 channels. Active TASK-1 channels maintain negative resting potential in hPASMCs. In hypoxia, the phospho-Src (active Src) is decreased. Closed TASK-1 leads to depolarisation and increased intracellular calcium level. Em: resting membrane potential. SrcTk: Src family tyrosine kinase; Kv: voltage-gated potassium currents; Kca: calcium-dependent potassium currents.

increase in the $\left[\mathrm{Ca}^{2+}\right]_{\mathrm{i}}$, results in increased proliferation and decreased apoptosis, ultimately contributing to the pathogenesis of pulmonary hypertension [16].

Depolarisation in hPASMCs, induced by the inhibition of potassium channels, is followed by influx of calcium through voltage-gated (L-type) calcium channels and results in increased pulmonary vascular resistance. Acute hypoxia causes vasoconstriction by several mechanisms. It depolarises PASMCs by inhibiting potassium channels, leading to calcium influx as described above [17], causes the release of calcium from the sarcoplasmic reticulum and subsequent repletion through storeoperated channels $[18,19]$, increases calcium influx into PASMCs through L-type calcium channels, independent of the membrane potential [20] and also promotes calcium sensitisation [21], thus increasing pulmonary vascular resistance [22]. Most of the calcium responsible for the increase in cytosolic calcium induced by hypoxia comes from outside the PASMC but some is released from internal stores, such as the sarcoplasmic reticulum [1]. It is likely that the reduction in the hypoxia-induced increase in calcium that is caused by PP2, siC-Src or siFyn (fig. 5) is secondary to the lack of hypoxic inhibition of potassium currents, because inhibition of potassium channels has already been caused by SrcTK inhibition. The hypoxic response is abolished in the presence of diminished SrcTK activity (figs 4 and 6).

Several studies have indicated that tyrosine kinases may act on potassium channels. However, these studies were carried out either on cell lines or in heterologous expression systems using a broad range inhibitors but without showing the physiological role of the findings $[7,23,24]$. We have previously shown that the background two pore domain TASK-1 channel sets the membrane potential in primary human PASMCs, and it can be modulated by PKA, PLC, PKC and AMPK pathways through serine-threonine phosphorylation by different agonists, such as endothelin [10], treprostinil [3], etc. Furthermore, hypoxia depolarises the membrane potential by reversibly inhibiting TASK-1 channels in hPASMCs [3]. In addition, $\mathrm{KCa}_{\mathrm{a}}$ and $\mathrm{K}_{\mathrm{v}}$ channels may also contribute to the membrane potential, particularly if they are stimulated by agents like cyclic AMP and cyclic GMP. Therefore, our results suggest that SrcTK activity is essential for the low physiological tone of PASMCs and, thus, for the low pulmonary vascular resistance.

The mechanism by which hypoxia inhibits these channels is currently unknown. However, in the present study, specific inhibition of endogenous SrcTK reduces TASK-1 current in hPASMC. Likewise, when hPASMC are dialysed with SrcTK activator, a significant increase in the TASK-1 current is observed, suggesting that TASK-1 current requires SrcTK activity, with dephosphorylation decreasing the current and phosphorylation increasing it. Several other potassium channels, including $\mathrm{KCa}$ and $\mathrm{K}_{\mathrm{v}}$, have previously been shown to be modulated by SrcTK-mediated tyrosine phosphorylation [25]. Our observations confirm this but demonstrate the critical role of ScrTK activity for the function of these channels and show that TASK-1 activity requires SrcTK activity.

In the present study, we demonstrated that SrcTK inhibition reduces TASK-1 current and plays a crucial role in the hypoxic inhibition of TASK-1 channels, probably through a reduction in phosphorylation of SrCTK at Tyr419 and dissociation of TASK-1 and SrcTK, although the molecular regulation of this link has yet to be determined (fig. 7).

Recent studies by KNOCK and co-workers [26, 27] describe that SrcTK inhibition by PP2 blunts hypoxia-induced pulmonary 
vasoconstriction and inhibits Rho kinase in rat pulmonary artery and isolated PASMCs. Their finding that PP2 reduces the hypoxia-induced increase in PASMC $\left[\mathrm{Ca}^{2+}\right]_{\mathrm{i}}$ is concordant with our observations. Given the inhibitory results of PP2 and the siRNAs on TASK-1, Kv and KCa currents that we describe here, and the similar effects of hypoxia on these currents, it makes sense that the SrcTK phosphorylation is inhibited in the sequence leading to potassium current inhibition. However, in their experiments, KNOCK and co-workers [26, 27] noted that hypoxia increased phosphorylation of SrcTK at Tyr419 rather than decreased it, as we also report here. The difference in these results may be due to differences in species, cell culture conditions, the number of cycles, and the severity, duration or time-point of the study under hypoxia.

The observed increase in Ppa following the inhibition of SrcTK by PP2 and dasatinib suggests a functional role for SrcTK in regulating pulmonary vascular tone. What this means for patients with severe pulmonary hypertension is not known. TUDER et al. [28] describe decreased levels of c-Src in 16 primary pulmonary hypertension (idiopathic pulmonary arterial hypertension (PAH) according to the current classification) patients. This would correspond to decreased potassium channel activity and might contribute to vasoconstriction and other pathological mechanisms leading to pulmonary hypertension. Herein, we report a reduced TASK-1, whole-cell potassium current and KCa current after c-Src inhibition in hPASMCs. This is in line with observations showing that reduced $\mathrm{K}_{v} 1.5$ activity leads to pulmonary hypertension [13]. In contrast, a recent study by COURBOULIN et al. [29] reports increased levels of phosphor-Src and total Src in lung samples from three PAH patients.

Recent clinical observations highlight the potential importance of tyrosine kinases in the pathophysiology of PAH. Chronic myeloid leukaemia (CML) is caused by a constitutively active BCR-ABL tyrosine kinase. Imatinib, which inhibits this kinase, is the first-line therapy for CML [30]. Imatinib is also an effective inhibitor of the platelet-derived growth factor receptor [31] and this is thought to be the reason why it may improve haemodynamics in some PAH patients [32]. Dasatinib is a tyrosine and a serine-threonine kinase inhibitor that is 325 times as potent as imatinib in the inhibition of BCR-ABL kinase in vitro, and induces higher rates of cytogenic response in CML [33]. However, dasatinib potently inhibits the Src family, which is probably the single most prominent dasatinib-targeted family of protein kinases, including c-Src and Fyn, and it has been reported to cause $\mathrm{PAH}[8,34,35]$. The $\mathrm{PAH}$ tends to resolve rapidly after discontinuation of dasatinib [34], suggesting that it may not be primarily due to marked cellular proliferation, but to chronic vasoconstriction. One can speculate that dasatinibinitiated pulmonary hypertension may relate to our finding that siRNA against c-Src and Fyn reduces potassium channel current and causes depolarisation of hPASMCs. It is clear that much work remains to be done to clarify the role of different kinases in the aetiology and therapy of PAH.

\section{Limitations of the study}

Although the present findings suggest that TASK-1 channels and SrcTK are co-localised in the plasma membrane of hPASMCs, which is further supported by co-immunoprecipitation studies, we cannot exclude that Src could also act indirectly on potassium channels via other Src downstream molecules. In addition, mechanism(s) involved in acute hypoxia-induced inhibition of potassium channels could be different than those involved with chronic exposure to hypoxia and/or dasatinib. Finally, changes in Ppa in the presence of PP2 and/or dasatinib might not be related to this precise proposed mechanism.

\section{Conclusion}

In conclusion, we demonstrate that SrcTK has an important role in the control of TASK-1 and other potassium channels, and that it sets the negative resting membrane potential in hPASMCs. The physiological relevance of this SrcTK and TASK-1 channel association is emphasised by the fact that the hypoxia-induced inhibition of TASK-1 current and the intracellular calcium rise are dependent on SrcTK. It is likely that a better description of the multifunctional role of SrCTK in regard to potassium channel regulation will facilitate our understanding of the pathophysiology of pulmonary hypertension.

\section{SUPPORT STATEMENT}

This study was funded in part by the Medical University of Graz (PhD programme in molecular medicine to C. Nagaraj). E.K. Weir is supported by VA Medical Research funding and RO1 HL 65322 (National Institutes of Health, Bethesda, MD, USA).

\section{STATEMENT OF INTEREST}

Statements of interest for H. Olschewski and A. Olschewski can be found at www.erj.ersjournals.com/site/misc/statements.xhtml

\section{ACKNOWLEDGEMENTS}

The excellent technical assistance of $\mathrm{M}$. Schloffer and P. Blümel (Experimental Anesthesiology, Dept of Anaesthesia and Intensive Care Medicine, Medical University of Graz, Graz, Austria) is greatly appreciated.

\section{REFERENCES}

1 Weir EK, Olschewski A. Role of ion channels in acute and chronic responses of the pulmonary vasculature to hypoxia. Cardiovasc Res 2006; 71: 630-641.

2 Gurney AM, Osipenko ON, MacMillan D, et al. Two-pore domain K channel, TASK-1, in pulmonary artery smooth muscle cells. Circ Res 2003; 93: 957-964.

3 Olschewski A, Li Y, Tang B, et al. Impact of TASK-1 in human pulmonary artery smooth muscle cells. Circ Res 2006; 98: 1072-1080.

4 Martin GS. The hunting of the Src. Nat Rev Mol Cell Biol 2001; 2: 467-475.

5 Yeatman TJ. A renaissance for SRC. Nat Rev Cancer 2004; 4: 470-480.

6 Dai S, Hall DD, Hell JW. Supramolecular assemblies and localized regulation of voltage-gated ion channels. Physiol Rev 2009; 89: 411-452.

7 Holmes TC, Fadool DA, Ren R, et al. Association of Src tyrosine kinase with a human potassium channel mediated by $\mathrm{SH} 3$ domain. Science 1996; 274: 2089-2091.

8 Montani D, Bergot E, Gunther S, et al. Pulmonary arterial hypertension in patients treated by dasatinib. Circulation 2012; 125: 2128-2137.

9 Kreneisz O, Benoit JP, Bayliss DA, et al. AMP-activated protein kinase inhibits TREK channels. J Physiol 2009; 587: 5819-5830.

10 Tang B, Li Y, Nagaraj C, et al. Endothelin-1 inhibits background two-pore domain channel TASK-1 in primary human pulmonary artery smooth muscle cells. Am J Respir Cell Mol Biol 2009; 41: 476-483. 
11 Czirjak G, Petheo GL, Spat A, et al. Inhibition of TASK-1 potassium channel by phospholipase C. Am J Physiol Cell Physiol 2001; 281: C700-C708.

12 Gurney AM, Osipenko ON, MacMillan D, et al. Potassium channels underlying the resting potential of pulmonary artery smooth muscle cells. Clin Exp Pharmacol Physiol 2002; 29: 330-333.

13 Yuan XJ, Wang J, Juhaszova $\mathrm{M}$, et al. Attenuated $\mathrm{K}^{+}$channel gene transcription in primary pulmonary hypertension. Lancet 1998; 351: 726-727.

14 Bonnet S, Rochefort G, Sutendra G, et al. The nuclear factor of activated $\mathrm{T}$ cells in pulmonary arterial hypertension can be therapeutically targeted. Proc Natl Acad Sci USA 2007; 104: 11418-11423.

15 Yuan JX, Aldinger AM, Juhaszova M, et al. Dysfunctional voltagegated $\mathrm{K}^{+}$channels in pulmonary artery smooth muscle cells of patients with primary pulmonary hypertension. Circulation 1998; 98: 1400-1406.

16 Mandegar M, Fung YC, Huang W, et al. Cellular and molecular mechanisms of pulmonary vascular remodeling: role in the development of pulmonary hypertension. Microvasc Res 2004; 68: 75-103.

17 Post JM, Hume JR, Archer SL, et al. Direct role for potassium channel inhibition in hypoxic pulmonary vasoconstriction. Am J Physiol 1992; 262: C882-C890.

18 Vadula MS, Kleinman JG, Madden JA. Effect of hypoxia and norepinephrine on cytoplasmic free $\mathrm{Ca}^{2+}$ in pulmonary and cerebral arterial myocytes. Am J Physiol 1993; 265: L591-L597.

19 Zhang S, Yuan JX, Barrett KE, et al. Role of $\mathrm{Na}^{+} / \mathrm{Ca}^{2+}$ exchange in regulating cytosolic $\mathrm{Ca}^{2+}$ in cultured human pulmonary artery smooth muscle cells. Am J Physiol Cell Physiol 2005; 288: C245-C252.

20 Del Valle-Rodriguez A, Lopez-Barneo J, Urena J. Ca ${ }^{2+}$ channelsarcoplasmic reticulum coupling: a mechanism of arterial myocyte contraction without $\mathrm{Ca}^{2+}$ influx. EMBO J 2003; 22: 4337-4345.

21 Robertson TP, Aaronson PI, Ward JP. $\mathrm{Ca}^{2+}$ sensitization during sustained hypoxic pulmonary vasoconstriction is endothelium dependent. Am J Physiol Lung Cell Mol Physiol 2003; 284: L1121-L1126.

22 Weir EK, Cabrera JA, Mahapatra S, et al. The role of ion channels in hypoxic pulmonary vasoconstriction. Adv Exp Med Biol 2010; 661: 3-14.

23 Holmes TC, Berman K, Swartz JE, et al. Expression of voltagegated potassium channels decreases cellular protein tyrosine phosphorylation. J Neurosci 1997; 17: 8964-8974.
24 Kirkegaard SS, Lambert IH, Gammeltoft S, et al. Activation of the TASK-2 channel after cell swelling is dependent on tyrosine phosphorylation. Am J Physiol Cell Physiol 2010; 299: C844-C853.

25 Alioua A, Mahajan A, Nishimaru K, et al. Coupling of c-Src to large conductance voltage- and $\mathrm{Ca}^{2+}$-activated $\mathrm{K}^{+}$channels as a new mechanism of agonist-induced vasoconstriction. Proc Natl Acad Sci USA 2002; 99: 14560-14565.

26 Knock GA, Snetkov VA, Shaifta Y, et al. Role of src-family kinases in hypoxic vasoconstriction of rat pulmonary artery. Cardiovasc Res 2008; 80: 453-462.

27 Knock GA, Shaifta Y, Snetkov VA, et al. Interaction between src family kinases and rho-kinase in agonist-induced $\mathrm{Ca}^{2+}$-sensitization of rat pulmonary artery. Cardiovasc Res 2008; 77: 570-579.

28 Tuder RM, Chacon M, Alger L, et al. Expression of angiogenesisrelated molecules in plexiform lesions in severe pulmonary hypertension: evidence for a process of disordered angiogenesis. J Pathol 2001; 195: 367-374.

29 Courboulin A, Paulin R, Giguère NJ, et al. Role for miR-204 in human pulmonary arterial hypertension. J Exp Med 2011; 208: 535-548.

30 Kantarjian H, Shah NP, Hochhaus A, et al. Dasatinib versus imatinib in newly diagnosed chronic-phase chronic myeloid leukemia. N Engl J Med 2010; 362: 2260-2270.

31 Schermuly RT, Dony E, Ghofrani HA, et al. Reversal of experimental pulmonary hypertension by PDGF inhibition. J Clin Invest 2005; 115: 2811-2821.

32 Ghofrani HA, Morrell NW, Hoeper MM, et al. Imatinib in pulmonary arterial hypertension patients with inadequate response to established therapy. Am J Respir Crit Care Med 2010; 37: 1104-1118.

33 Rix U, Hantschel O, Durnberger G, et al. Chemical proteomic profiles of the BCR-ABL inhibitors imatinib, nilotinib, and dasatinib reveal novel kinase and nonkinase targets. Blood 2007; 110: 4055-4063.

34 Dumitrescu D, Seck C, ten Freyhans H, et al. Fully reversible pulmonary arterial hypertension associated with dasatinib treatment for chronic myeloid leukaemia. Eur Respir J 2011; 38: 218-220.

35 Mattei D, Feola M, Orzan F, et al. Reversible dasatinib-induced pulmonary arterial hypertension and right ventricle failure in a previously allografted CML patient. Bone Marrow Transplant 2009; 43: 967-968. 